\title{
Motion fading and the motion aftereffect share a common process of neural adaptation
}

\author{
P.-J. HSIEH AND P. U. TSE \\ Dartmouth College, Hanover, New Hampshire
}

\begin{abstract}
After prolonged viewing of a slowly drifting or rotating pattern under strict fixation, the pattern appears to slow down and then momentarily stop. Here, we show that this motion fading occurs not only for slowly moving stimuli, but also for stimuli moving at high speed; after prolonged viewing of high-speed stimuli, the stimuli appear to slow down but not to stop. We report psychophysical evidence that the same neural adaptation process likely gives rise to motion fading and to the motion aftereffect.
\end{abstract}

After prolonged viewing of a slowly drifting or rotating pattern under strict fixation, the pattern appears to slow down and then momentarily stop, even though the stationary form of the pattern remains visible. This motion fading has been reported to occur over slowly rotating gratings and spinning sector disks (Campbell \& Maffei, 1979, 1981; Cohen, 1965; Hunzelmann \& Spillmann, 1984; Lichtenstein, 1963; MacKay, 1982). Several factors, including retinal eccentricity, number of sectors, speed of rotation (Hunzelmann \& Spillmann, 1984), and salience of trackable features (such as corners; Hsieh \& Tse, 2007), have been shown to affect the time required for motion fading.

According to the motion aftereffect (MAE) hypothesis tested here, motion fading arises because of adaptation among cortical motion-tuned neurons that are the same as those that underlie the well-known MAE, where illusory motion is perceived to occur over a stationary object or image following prolonged exposure to visual motion (Wohlgemuth, 1911). For example, it has been shown that the MAE is based on neural adaptation (i.e., modulation of gain control) near or at the input of MT (Kohn \& Movshon, 2003). Van de Grind, van der Smagt, and Verstraten (2004; see also van de Grind, Lankheet, \& Tao, 2003) showed how such adaptation might occur, on the basis of the gain-control model of Grunewald and Lankheet (1996). Moreover, Kohn and Movshon showed that the MAE mechanism is realized in the motion pathway, not the form pathway, which is consistent with the phenomenology of motion fading, where only the motion component appears to vanish from consciousness, while the form component remains visible. Together, these findings are consistent with the hypothesis that adaptation among motion-tuned neurons underlies motion fading.

In this article, we first will examine whether the motionfading effect can occur over stimuli at high speed. To our knowledge, motion fading over stimuli moving at high speeds has not been carefully examined. However, it has been shown that a flickering spot/grating presented in the peripheral visual field appears to lose contrast and stop flickering (Anstis, 1996; Frome, MacLeod, Buck, \& Williams, 1981; Hammett \& Smith, 1990; Harris, Calvert, \& Snelgar, 1990; Schieting \& Spillmann, 1987). Given that moving and flickering stimuli share similar spatiotemporal properties, it is reasonable to hypothesize that motion fading can also occur over stimuli moving at high speed. This possibility was examined in Experiment 1. In Experiment 2, the degree to which motion fading occurs was mapped across the visual field. In Experiment 3, we measured motion fading as a function of stimulus spatial frequency. In Experiment 4, we tested whether the subjective decrement of perceived luminance in motion fading is correlated with the subjective decrement of perceived speed. In Experiments 5 and 6, we directly tested the MAE hypotheses stated above.

\section{EXPERIMENT 1 Factors Affecting Motion Fading}

In Experiment 1, rather than using a slowly moving target, as has been tested previously (Campbell \& Maffei, 1979, 1981; Cohen, 1965; Hsieh \& Tse, 2007; Hunzelmann \& Spillmann, 1984; Lichtenstein, 1963; MacKay, 1982), we examined whether motion fading can occur over stimuli moving at high speed. We measured the perceived speed of the adapted motion pattern by asking subjects to adjust the speed on a nonadapted motion pattern presented in the mirror-opposite location (relative to the vertical axis) of the adapted motion pattern (Figure 1A). To investigate some possible factors affecting perceived speed during motion fading, we systematically changed the speed and luminance of the target motion pattern and measured the difference between the veridical and the perceived speeds. 
A

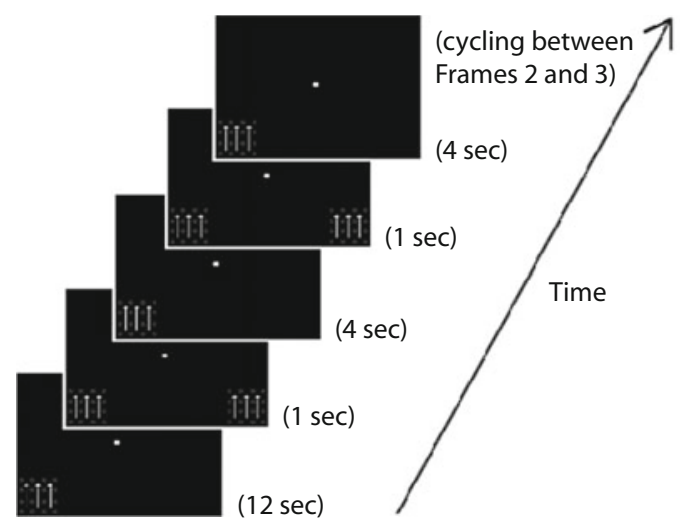

B

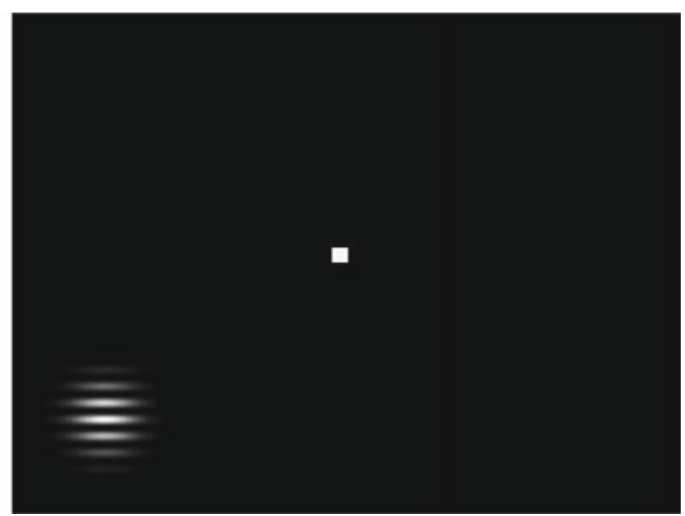

Figure 1. Example stimuli. (A) An example trial of Experiment 1. The white arrows were not present in the actual stimulus. (B) Example stimuli used in Experiment 3.

\section{Method}

Observers. There were 6 subjects in each experiment. Four of the subjects (including one author) participated in all the experiments. All of them had normal or corrected-to-normal vision. Before each experiment, the subjects practiced several training trials until they were accustomed to the experimental procedure and were capable of fixating while conducting hand movements.

Stimuli and Procedure. The stimulus configuration and experimental procedure used in Experiment 1 are shown in Figure 1A. The fixation spot was a yellow square (luminance, $128 \mathrm{~cd} / \mathrm{m}^{2} ; \mathrm{CIE}$, $x=0.429, y=0.497$; measured using a Minolta 100LS colorimeter) that subtended $0.05^{\circ}$ of visual angle. The default target stimulus was composed of 16 blue squares (luminance, $1.9 \mathrm{~cd} / \mathrm{m}^{2}$; CIE, $x=$ $0.141, y=0.071)$, each subtending $0.4^{\circ}$ of visual angle in width and height. The distance between each square (center to center) was $1.0^{\circ}$ of visual angle. All the squares moved upward at a constant rate $\left(1.6^{\circ} / \mathrm{sec}\right)$ within a $4.3^{\circ} \times 4.3^{\circ}$ window on a black $\left(\sim 0 \mathrm{~cd} / \mathrm{m}^{2}\right)$ background. When the squares left this window, they disappeared. The target stimulus containing moving squares was presented at either the upper left corner $\left(16^{\circ}\right.$ left of the vertical midline and $10^{\circ}$ above the horizontal midline) or lower left corner $\left(16^{\circ}\right.$ left of the vertical midline and $10^{\circ}$ below the horizontal midline). Another stimulus (the comparison stimulus) containing the identical motion pattern was presented at the position mirror-opposite that of the target stimulus (relative to the vertical axis). The target stimulus was continuously present on the screen. Nine seconds after target stimulus onset, the comparison stimulus was presented for $1 \mathrm{sec}$ and then was removed from the screen for $3 \mathrm{sec}$ (cycling until the subjects made a response) to prevent the induction of the motion-slowing effect that we wished to characterize.

In Experiment 1A (speed-changing experiment), the subjects were presented with the default stimuli above and were required to adjust the speed of the comparison stimulus to match the corresponding properties of the target stimulus by pressing two buttons: One increased and the other decreased the speed of the comparison stimulus. The luminance of the target stimulus and the comparison stimulus remained the same $\left(1.9 \mathrm{~cd} / \mathrm{m}^{2}\right)$, whereas the speed of the target stimulus was randomly assigned to be $1.15^{\circ}, 1.62^{\circ}, 2.30^{\circ}$, $3.29^{\circ}, 3.68^{\circ}, 4.6^{\circ}$, or $5.75^{\circ} / \mathrm{sec}$. The initial speed of the comparison stimulus was randomly assigned to be either 1.2 times (i.e., faster than) or 0.3 times (i.e., slower than) the speed of the target stimulus. In Experiment 1B (luminance-changing experiment), the speed of the target remained the same $(3.29 \% \mathrm{sec})$, whereas the luminance of the target stimulus was randomly assigned to be $0.1,0.4,0.9,1.4$, $2.1,3.0$, or $4.0 \mathrm{~cd} / \mathrm{m}^{2}$. The luminance of the comparison stimulus remained the same, and the initial speed of the comparison stimulus was randomly assigned to be either 1.2 times or 0.3 times the speed of the target stimulus. All possibilities were pseudorandomly counterbalanced across 28 trials. The subjects were required to rest after each trial until any possible afterimage had disappeared.

All the stimuli in the present study were viewed with both eyes. The total size of the visual field was $40 \times 30 \mathrm{~cm}$, viewed from a distance of $57 \mathrm{~cm}$. The subjects had their chin in a chinrest. The visual stimulator was a 2-GHz Dell workstation running Windows 2000. The stimuli were presented on a 20-in. Mitsubishi CRT gammacorrected monitor with $1,600 \times 1,200$ pixel resolution and an $85-\mathrm{Hz}$ frame rate. Eye movements were monitored using a head-mounted eyetracker (Eyelink2; SR Research, Ontario, Canada). Trials during which the subject's monitored left eye was outside a fixation window with a $1.5^{\circ}$ radius for more than $1 \mathrm{sec}$ were forced to stop and restart after the subject regained fixation. Thus, all the data reported here were carried out under conditions of fixation.

\section{Results}

Figure 2A shows the results for the 4 subjects in Experiment $1 \mathrm{~A}$, which demonstrate the effect of motion fading over stimuli with different speeds (Figure 2B shows the mean across subjects). Among the seven tested speeds, the degree of perceived motion slowing was positively correlated with the veridical speed of the motion pattern: The higher the veridical speed, the more the perceived speed decreased. A repeated measures ANOVA was performed to show that there was a difference across the seven tested speeds $[F(6,30)=46.045, p<.001]$; a repeated measures ANOVA with a linear contrast was then carried out and showed that there was a parametric variation of perceived speeds with increases in the tested speeds $[F(1,5)=97.904, p<.001]$. Figure $2 \mathrm{C}$ shows the same data for the 4 subjects (Figure 2D shows the mean across subjects), but plotted with the percentage of decrease of speed as a function of veridical speed. The results show that the percentage of decrease of speed was constant across conditions.

In contrast, Figures $3 \mathrm{~A}$ and $3 \mathrm{~B}$ show that, among the seven tested luminances, the veridical luminance of the motion pattern did not affect the degree of perceived motion slowing across different luminances [repeated measures ANOVA: $F(6,30)=1.617, p=.177]$. 

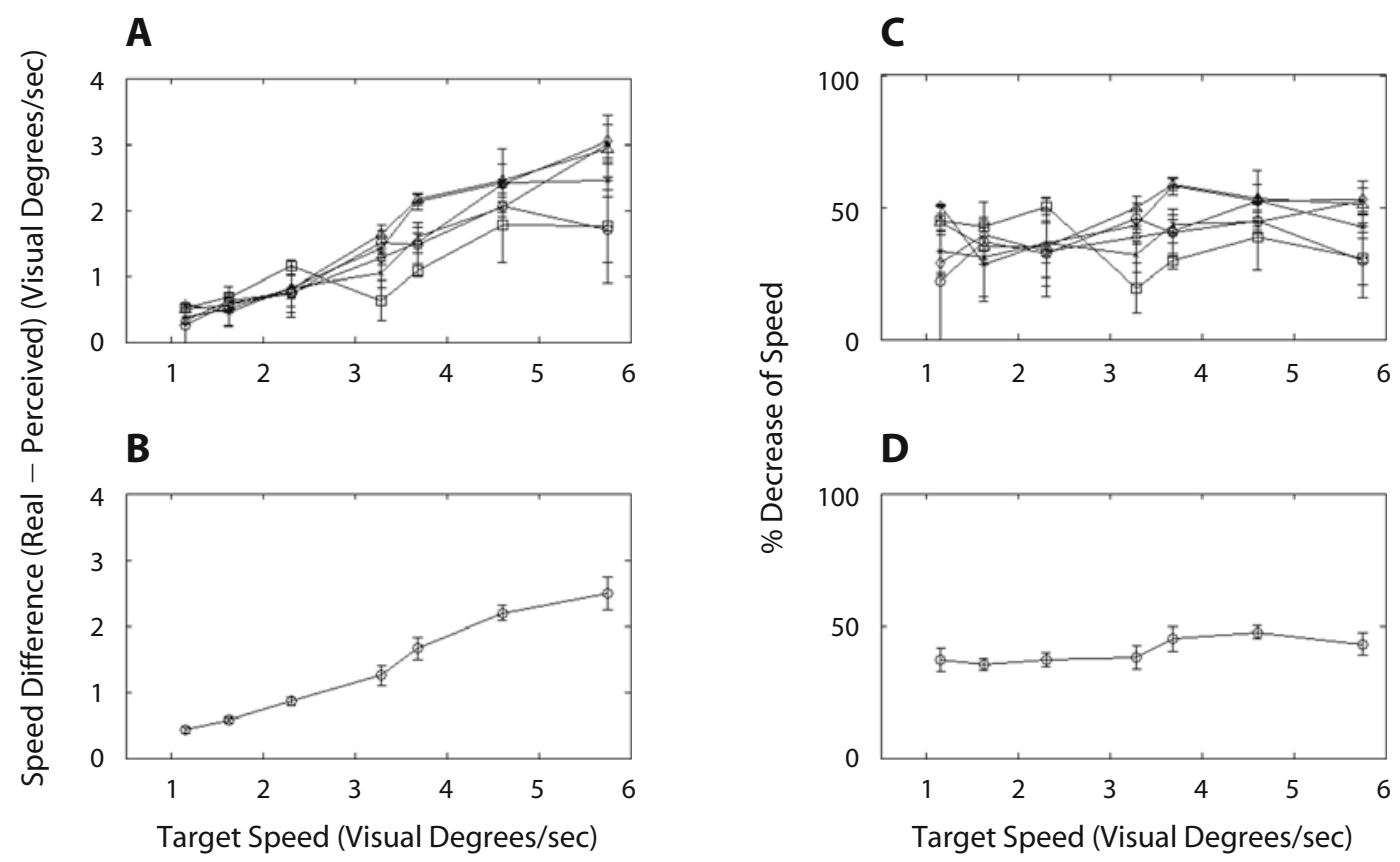

Figure 2. (A) Perceived decrements of speed for 4 subjects. The amount of decrement of the perceived speed was positively correlated with the veridical speed of the tested motion pattern. The higher the veridical speed, the more the perceived speed decreased. (B) Perceived decrements of speed averaged across 4 subjects. (C) Percentage of decrease of speed was a constant of about $45 \%$ of the veridical speed of the tested motion pattern. (D) Percentage of decrease of speed averaged across 4 subjects. Error bars in panels B and D indicate standard errors of the means across subjects.

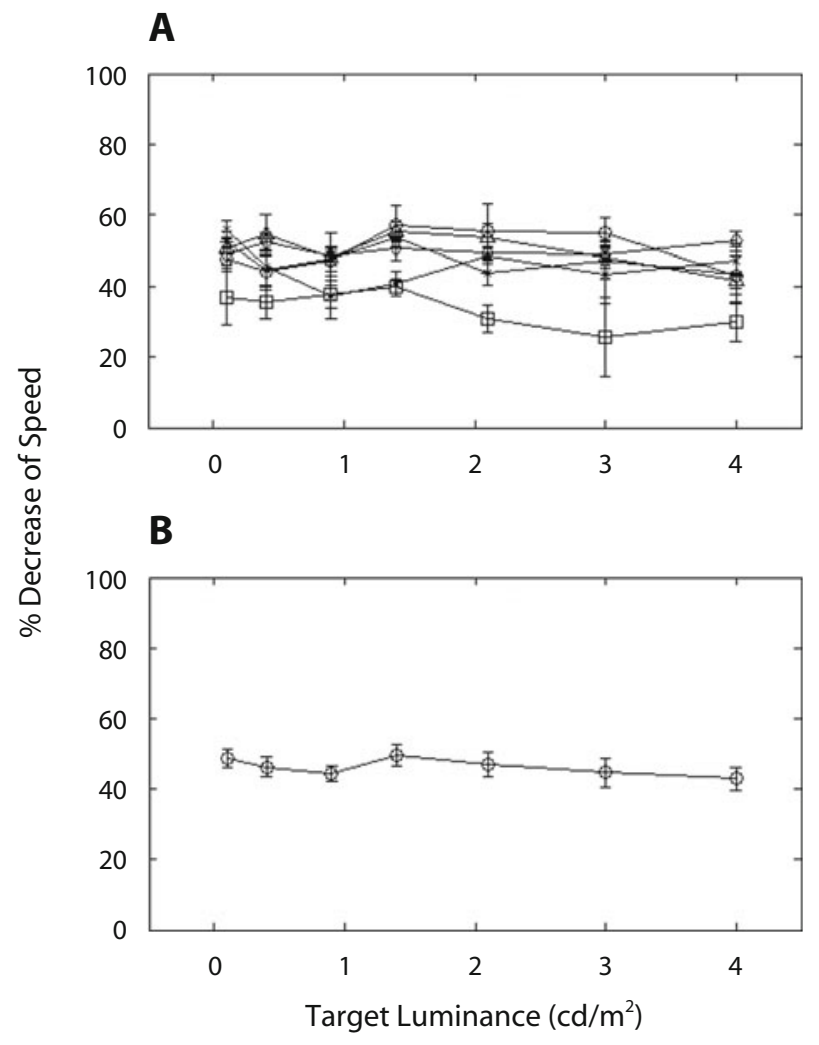

Figure 3. (A) Percentage of decrease of speed for 4 subjects was a constant of about $45 \%$ at all tested luminances. (B) Percentage of decrease of speed averaged across 4 subjects. Error bars in panel $B$ indicate standard errors of the means across subjects. 


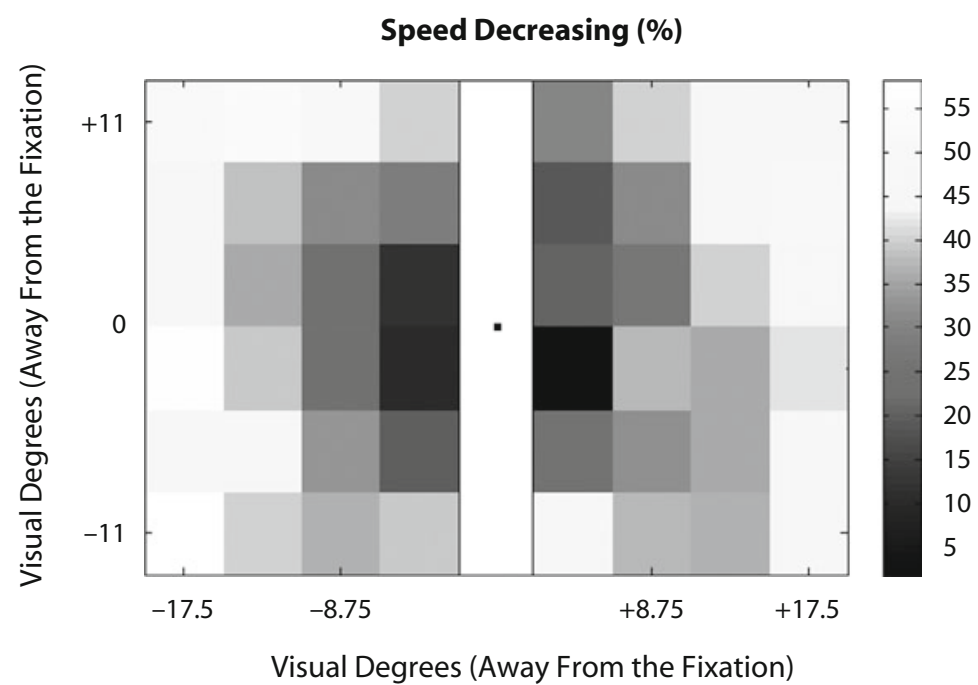

\begin{abstract}
Figure 4. The perceived speed of the motion pattern appeared to slow down by as much as $57 \%$ when the stimulus was located peripherally. In the central visual field, motion fading was less effective. Note that the vertical meridian is not mapped, because no mirror positions exist for the vertical meridian. Such positions were necessary with our method of measurement.
\end{abstract}

\section{EXPERIMENT 2 \\ Motion Fading As a Function of Stimulus Position Across Visual Field}

In Experiment 2, we systematically changed the position of the moving stimuli and measured the time to stop (TTS).

\section{Method \\ Stimuli and Procedure. In Experiment 2 (mapping experiment), the stimuli and procedure were identical to those in Experiment 1, except that the visual field on the screen was divided into 48 abutting virtual squares $\left(4.3^{\circ} \times 4.3^{\circ}\right)$. In each trial, the stimulus (moving at $1.6^{\circ} / \mathrm{sec}$ with a luminance value of $1.9 \mathrm{~cd} / \mathrm{m}^{2}$ ) was presented in 1 of the 48 locations. To avoid possible interference of afterimages across trials, testing locations were pseudorandomized across 48 trials so that the same location (or its mirror location) would not be tested consecutively. The subjects were required to rest after each trial until the afterimage had disappeared.}

\section{Results}

The results from Experiment 2 showed the strength of the motion-fading effect as a function of stimulus location across the whole visual field, except for the vertical midline, because of the impossibility of having mirror-opposite positions there (Figure 4). The results showed that the degree of perceived motion slowing was maximal when the motion pattern was located peripherally, where the perceived speed of the motion pattern could decrease by as much as $\sim 57 \%$ relative to the veridical speed. Perceived speed hardly slowed at all when the stimulus was located centrally.

\section{EXPERIMENT 3 \\ Motion Fading As a Function of Stimulus Spatial Frequency}

In Experiment 3, we systematically changed the spatial frequency and measured the TTS.

\section{Method}

Stimuli and Procedure. The stimulus configuration for Experiment 3 is shown in Figure 1B. The target stimulus was a Gabor (maximum luminance, $138 \mathrm{~cd} / \mathrm{m}^{2}$; minimum luminance, $\sim 0 \mathrm{~cd} / \mathrm{m}^{2}$ ), subtending $5^{\circ}$ of visual angle in width and height. In each trial, the Gabor was randomly assigned to be one of the following spatial frequencies $(0.500,0.63,0.77,0.91,1.111,1.25$, or $1.43 \mathrm{cycles} / \mathrm{deg})$, and the speed of the local drifting sine wave motion was $0.31 \% \mathrm{sec}$. The stimulus was always present on the screen at one of two possible locations: (1) $12.5^{\circ}$ left of the vertical midline and $7.5^{\circ}$ below the horizontal midline, or (2) $12.5^{\circ}$ right of the vertical midline and $7.5^{\circ}$ below the horizontal midline. The subjects were required to press a button when the motion first appeared to fully stop. All conditions were pseudorandomly counterbalanced across 28 trials (a block). The subjects participated in one to two blocks, and trials across blocks were pooled together.

\section{Results}

In Experiment 3, we measured the TTS by systematically changing the spatial frequency of a locally drifting Gabor. Figure 5 shows that TTS increased as the spatial frequency increased [repeated measures ANOVA, $F(6,30)=9.879, p<.001$; repeated measures ANOVA with a linear contrast, $F(1,5)=19.822, p=.007]$.

\section{EXPERIMENT 4 \\ Subjective Change of Perceived Luminance in Motion Fading and Its Influence}

In Experiment 4, we measured perceived luminances in motion fading. We also measured the lowest luminance that a stimulus needs in order to be perceived as moving by asking the subjects to lower the luminance of a constantly moving stimulus until it appeared to stop.

\section{Method}

Stimuli and Procedure. The target stimulus was one solid white square, subtending $3.4^{\circ}$ in height and width and rotating continuously counterclockwise with respect to the square center (not around the 
A

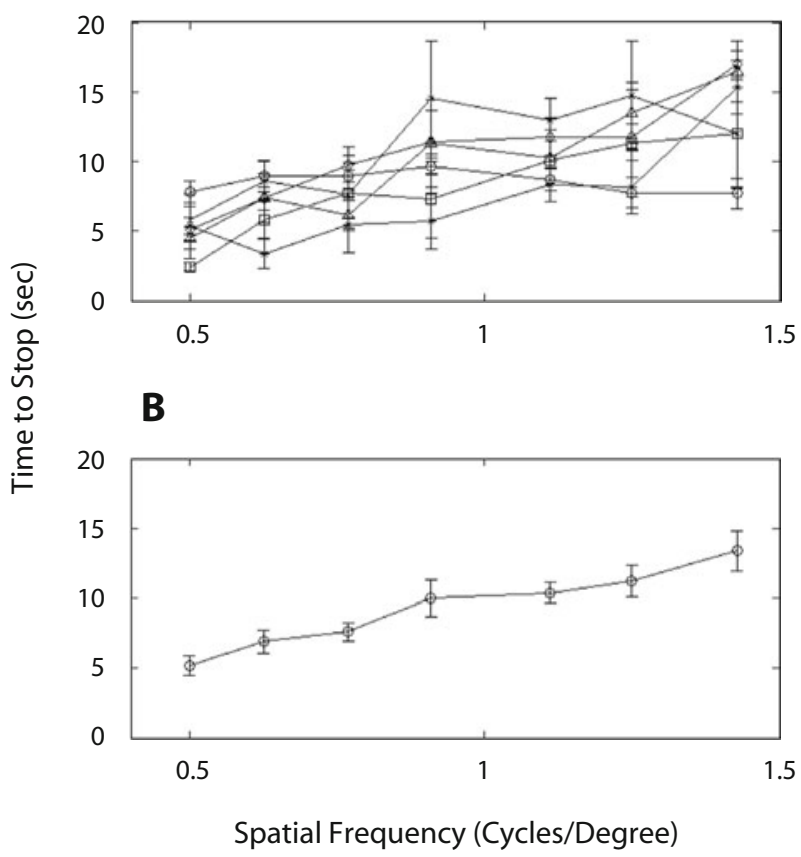

Figure 5. Time to stop (TTS) during motion fading as a function of tested spatial frequency in the target motion pattern. (A) TTS increased as the spatial frequency increased (individual subject data). (B) TTS averaged across subjects.

screen center) at the speed of $0.0235 \mathrm{rps}$. The target stimulus was present at one of two possible locations: (1) $12.5^{\circ}$ left of the vertical midline and $7.5^{\circ}$ below the horizontal midline, or (2) $12.5^{\circ}$ right of the vertical midline and $7.5^{\circ}$ below the horizontal midline. The luminance of the target stimulus was randomly assigned to be $25.1,65$, or $130 \mathrm{~cd} / \mathrm{m}^{2}$. Another square identical to the target stimulus was presented at the position mirror-opposite that of the target stimulus (relative to the vertical axis). The target stimulus was continuously present on the screen. The comparison stimulus was presented for $1 \mathrm{sec}$ and then was removed from the screen for $1 \mathrm{sec}$ repeatedly to prevent the induction of the motion fading effect that we wished to characterize. The subjects were required to adjust the luminance of the comparison square to match that of the target square that underwent motion fading.

The stimuli and procedure in Experiment $4 \mathrm{~B}$ were similar to those in Experiment 4A, except that there was no comparison stimulus. The target square was presented for $1 \mathrm{sec}$ and then was removed from the screen for $1 \mathrm{sec}$ repeatedly to prevent the induction of the very motion-fading effect that we wished to characterize. The luminance of the target stimulus was randomly assigned to be $25.1,65$, or $130 \mathrm{~cd} / \mathrm{m}^{2}$. The subjects were required to lower the luminance of the target square until it appeared to stop.

\section{Results}

Figure 6 shows that the perceived luminances dropped to about $36 \%$ of the veridical luminance for each target luminance in every condition (red curve), relative to the original target luminances (blue curve). Figure 6 also shows that the perceived luminances in every condition are much higher than the lowest luminance that a stimulus needs to have in order to be perceived as moving (the black curve). A repeated measures ANOVA was carried out and showed that the perceived luminances were significantly different from the lowest luminances that a stimulus needs to have in order to be perceived as moving $[F(1,5)=27.227, p=$ $.003]$. This result suggests that motion fading does not simply arise because of a decrease in luminance contrast. If the perceived decrease of luminance contrast after adaptation were the only cause of motion fading, all of the perceived luminances would have dropped to the lowest luminance that a stimulus needs to have to be perceived as moving.

\section{EXPERIMENT 5 Motion Fading Shares Common Neural Adaptation With Motion Aftereffect}

In order to further examine whether motion fading and the MAE are due to a common underlying mechanism, the two effects were pitted against each other in Experi-
A

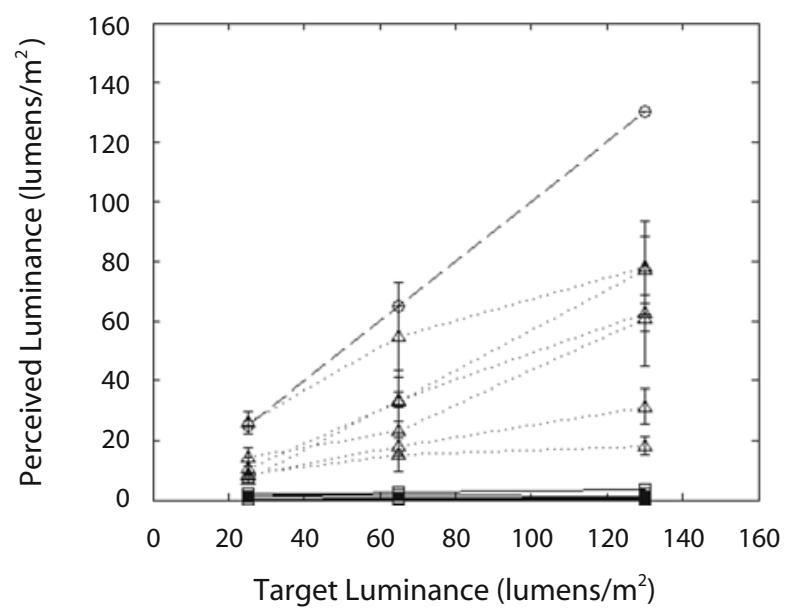

B

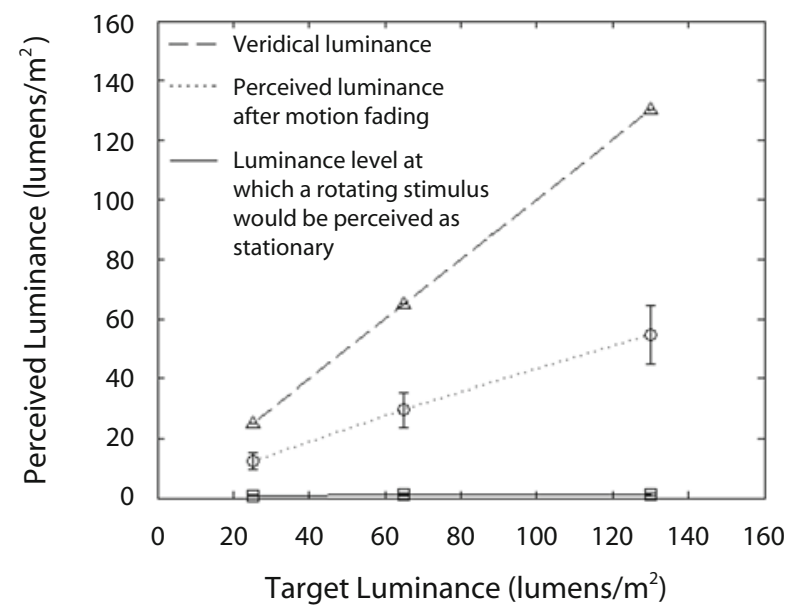

Figure 6. Perceived luminances after motion fading. The dashed line shows the initial target luminances (veridical luminance). The dotted lines show perceived luminances after motion fading. The solid lines show the luminance level at which a rotating stimulus would appear to be stationary. (A) Data from individual subjects. (B) Average data. Error bars in panel B indicate standard errors of the means across subjects. 
ment 5. The subjects were first adapted to a counterclockwise rotating pattern that generated the MAE and then were presented with a counterclockwise target (a square) rotating at the same angular velocity. The time it took for the target square to fade was measured. If the MAE and motion fading are due to a common underlying mechanism, such as the adaptation of motion-tuned cells, motion fading should occur more quickly the stronger the adaptation to a motion pattern that generates the MAE. If motion fading is not due to the neural adaptation that causes the MAE, adapting to a motion pattern that generates the MAE should not facilitate or hinder motion fading on a subsequent motion pattern.

\section{Method}

Stimuli and Procedure. The stimulus configuration for Experiment 5 is shown in Figure 7A. The fixation spot was a yellow square that subtended $0.05^{\circ}$ of visual angle. The target stimulus was one

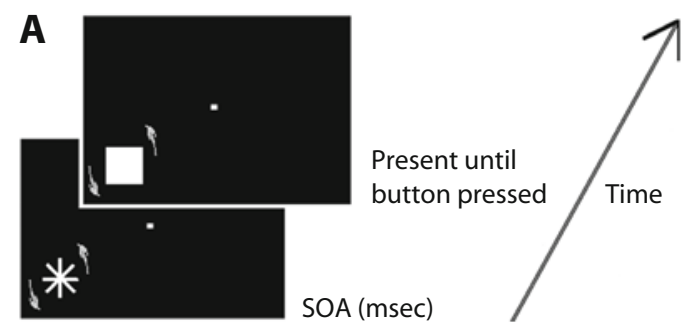

B

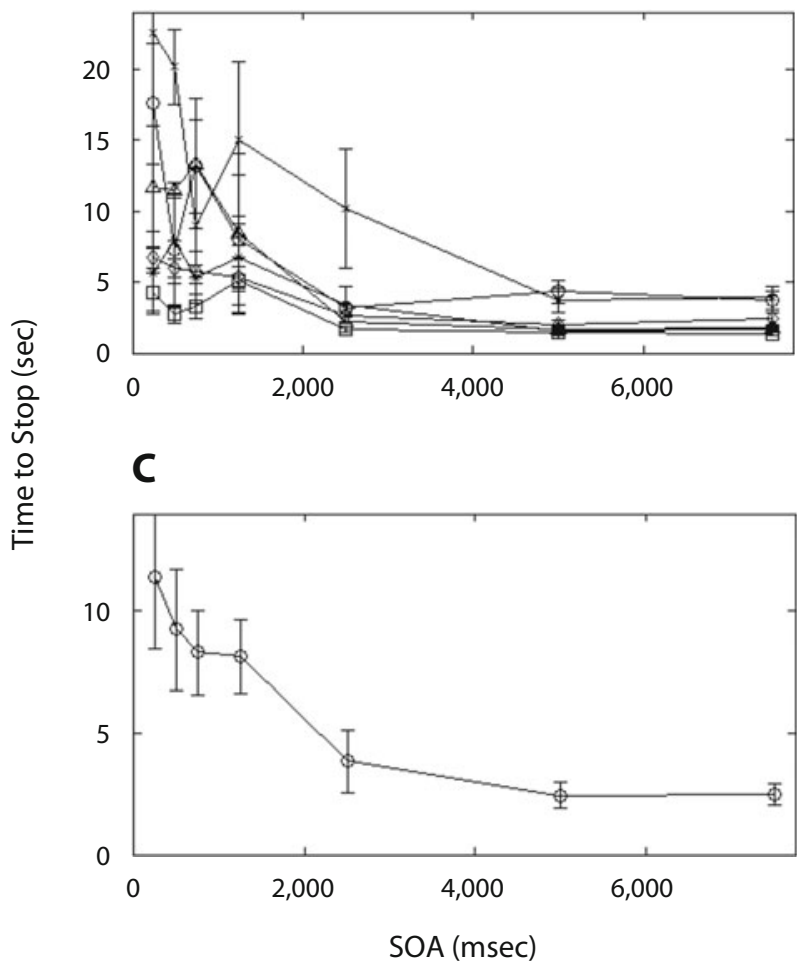

Figure 7. (A) Example stimuli of Experiment 5. (B) Results of Experiment 5. As the time (stimulus onset asynchrony [SOA]) of adapting to the radial pattern increased, the time to stop decreased. solid white (luminance: $65 \mathrm{~cd} / \mathrm{m}^{2}$ ) square, subtending $3.4^{\circ}$ in height and width, rotating at a constant angular velocity $(0.013 \mathrm{rps})$. The target stimulus was presented at one of the four possible positions: (1) $12.5^{\circ}$ left of the vertical midline and $7.5^{\circ}$ above the horizontal midline, (2) $12.5^{\circ}$ left of the vertical midline and $7.5^{\circ}$ below the horizontal midline, (3) $12.5^{\circ}$ right of the vertical midline and $7.5^{\circ}$ above the horizontal midline, and (4) $12.5^{\circ}$ right of the vertical midline and $7.5^{\circ}$ below the horizontal midline. During each trial, the rotating target stimulus was preceded by a rotating radial pattern with a certain stimulus onset asynchrony (SOA): 250, 500, 750, 1,250, $2,500,5,000$, or $7,500 \mathrm{msec}$. There was no interstimulus interval; the target square appeared immediately after the offset of the rotating radial pattern. The rotating radial pattern was composed of four white (luminance: $65 \mathrm{~cd} / \mathrm{m}^{2}$ ) bars, subtending $4.5^{\circ}$ in height and $0.15^{\circ}$ in width. The four bars were centered at the same location as the target square, equally spaced on a $360^{\circ}$ imaginary disk and always rotating at a constant angular velocity $(0.021 \mathrm{rps})$. The subjects were asked to press a button to indicate the TTS of the rotating square.

\section{Results}

The results in Figures 7B and 7C show that, in Experiment 5 , TTS decreased as the adaptation time (SOA) increased. A repeated measures ANOVA was carried out and showed that TTS decreased as the SOA increased [repeated measures ANOVA, $F(6,30)=8.246, p<.001$; repeated measures ANOVA with a linear contrast, $F(1,5)=$ $15.029, p=.012]$. This result shows that motion fading occurs more quickly after a stronger adaptation to a motion pattern that generates the MAE. In other words, motion fading is facilitated by longer adaptation to a rotating radial pattern that strengthens the MAE. Therefore, it is likely that motion fading arises, at least in part, because of activity within the same neural populations that undergo the MAE and, by extension, arises via the same neural adaptation that underlies the MAE.

\section{EXPERIMENT 6 \\ Strength of the MAE Increases and Asymptotes As the Duration of Time That a Stimulus Is Viewed Increases}

In Experiment 6, we further tested whether motion fading and the MAE are due to a common underlying mechanism. In this experiment, a counterclockwise rotating pattern was presented for different durations of time, after which the pattern would physically stop and the duration of the MAE was measured. If motion fading arises because of the same neural adaptation as that underlying the MAE, we would predict that (1) the duration of MAE should be positively correlated with the duration of time that the counterclockwise rotating pattern was presented, and (2) for cases in which the counterclockwise rotating pattern had appeared to stop, when the pattern in fact physically stopped subsequent to subjective stopping, the duration of the MAE should be constant. This would presumably occur because the level of neuronal adaptation would have reached a maximum at which no motion was encoded (i.e., because the counterclockwise rotating pattern appeared to be stopped).

\section{Method}

Stimuli and Procedure. The stimulus configuration for Experiment 6 is shown in Figure 8A. All the procedures were identical to those in Experiment 5, except for the following differences. The 
target stimulus was composed of 12 white (luminance: $65 \mathrm{~cd} / \mathrm{m}^{2}$ ) disks, subtending $0.2^{\circ}$ of visual angle in diameter. The 12 disks were arranged to lie at three eccentricities from the center of the stimulus. The inner (middle, outer) "ring" was composed of 4 disks, located $\pm 0.4^{\circ}\left( \pm 1.4^{\circ}, \pm 2.4^{\circ}\right)$ horizontally and $\pm 0.4^{\circ}\left( \pm 1.4^{\circ}, \pm 2.4^{\circ}\right)$ vertically away from the center of the stimulus. The disks in the middle ring were aligned between the inner and outer layers to form the grouped shape of a cross. The rotating pattern was centered $12.5^{\circ}$ left of the vertical midline and $7.25^{\circ}$ below the horizontal midline and was always rotating counterclockwise at a constant speed (0.02 rps).

There were two parts to Experiment 6. In Experiment 6A, there were 10 trials. On each trial, the subjects were asked to fixate carefully on the fixation spot and to press a button to indicate when the rotating pattern appeared to stop. After each trial, the subjects were required to rest until the MAE disappeared and then were allowed to start the next trial.

There were 30 trials in Experiment 6B. On each trial, the subjects were adapted to the rotating pattern for a different duration of time $(0.117,0.938,1.875,3.75,7.5,15,18,24,30$, or $36 \mathrm{sec})$. After adaptation, the rotating pattern would physically stop, and the fixation spot would change colors from yellow to green (luminance, $100 \mathrm{~cd} / \mathrm{m}^{2}$; CIE, $x=0.296, y=0.603$ ), which served as a cue for the subjects
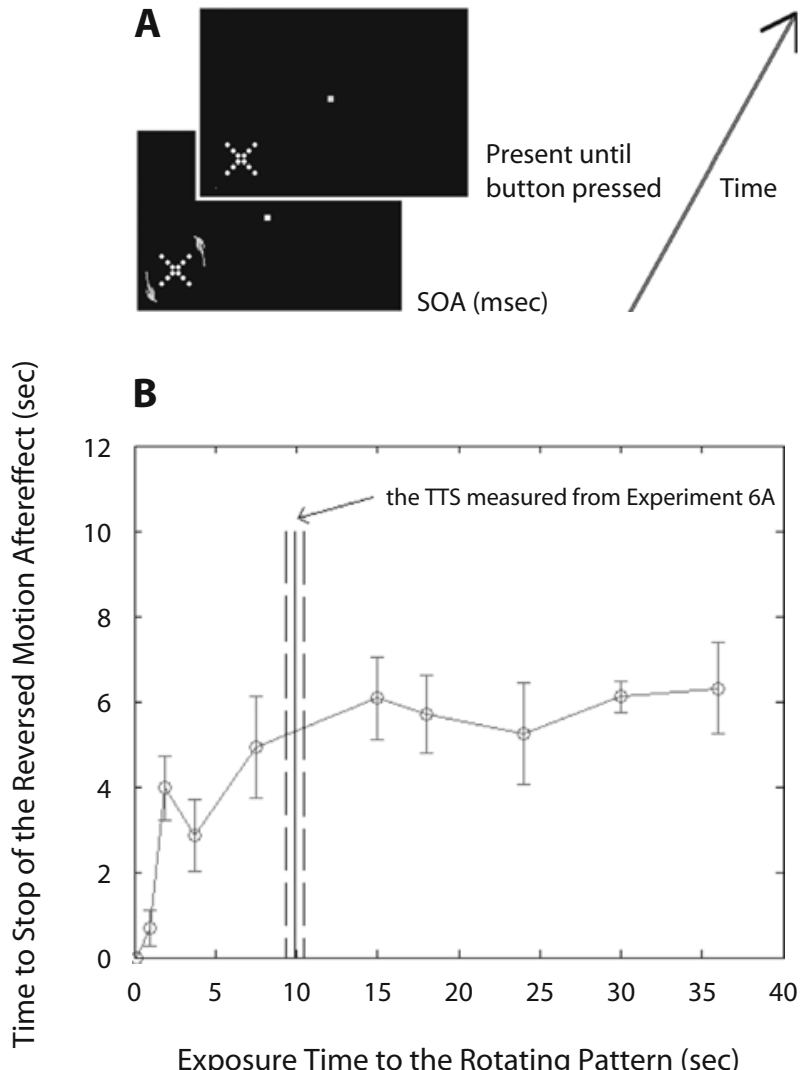

Figure 8. (A) Example stimuli of Experiment 6. (B) Results of Experiment 6. The gray curve shows that the duration of the motion of aftereffect (MAE) was positively correlated with the duration for which the counterclockwise rotating pattern was presented. The duration of the MAE asymptotes when the rotating pattern was presented for longer than about $10-15 \mathrm{sec}$, which is very close to the time it takes for the original rotating pattern to fade (superimposed on the figure as a vertical black line). (Note that the two dashed lines indicate the standard error for the black line across subjects.) SOA, stimulus onset asynchrony; TTS, time to stop. to respond. After the fixation point color changed, the subjects were required to indicate when the MAE disappeared by pressing a button. If the subjects did not perceive any MAE, they pressed a different button, and the duration of MAE would be recorded as 0 .

\section{Results}

The results in Figure 8B show that, in Experiment 6B, the duration of the MAE was positively correlated with the duration for which the counterclockwise rotating pattern was presented. A repeated measures ANOVA was carried out and showed that there was a significant difference across conditions $[F(9,45)=11.700, p<.001]$. The duration of the MAE asymptotes when the rotating pattern was presented for longer than about $10-15 \mathrm{sec}$, which is very close to the time it takes for the rotating pattern to fade (the results from Experiment 6A, superimposed on Figure $8 \mathrm{~B}$ as a blue line). Therefore, when the counterclockwise rotating pattern was presented for too long, so that the rotating motion signal subjectively fades, the duration of its MAE asymptotes at a constant duration (about $10-15 \mathrm{sec}$ ). This is presumably because the level of neuronal adaptation reaches its maximum after the rotating motion signal appears to stop. Together, the results from Experiments 5 and 6 suggest that motion fading arises at least in part because of the same neural adaptation as that underlying the MAE and, indeed, arises from activity within the same population of cells.

\section{DISCUSSION}

The goal of the present research was to determine the neural mechanisms underlying motion fading. We found that motion fading not only occurs over slowly moving stimuli, as has been reported in the past, but also can be observed over stimuli moving at high speeds. After prolonged viewing of stimuli moving at high speeds, the stimuli appear to slow down but not to stop. Our results show that the degree of perceived motion slowing is maximal when the motion pattern is located peripherally and hardly slows at all when the stimulus is located centrally. This result is consistent with previous findings that the magnitude of the MAE for small-field translational motions and even complex motions increases with eccentricity (Castet, Keeble, \& Verstraten, 2002; Murakami \& Shimojo, 1995; Price, Greenwood, \& Ibbotson, 2004; Wright, 1986). This increase in magnitude with eccentricity may occur for both the MAE and motion fading, because the preponderance of magnocellular cells increases with distance from the fovea and it is magnocellular processing that primarily underlies motion processing. In addition, it has been suggested that two populations of motion detectors have independent involvement in generating the MAE: (1) high-speed motion sensors absent at the fovea and (2) low-speed motion sensors absent at greater eccentricities (van de Grind, Koenderink, \& van Doorn, 1986; van de Grind, van Hof, van der Smagt, \& Verstraten, 2001; van der Smagt, Verstraten, \& van de Grind, 1999; Verstraten, van der Smagt, Fredericksen, \& van de Grind, 1999; Verstraten, van der Smagt, \& van de Grind, 1998). These findings might be able to explain why slow motion is seen as stopped mo- 
tion at higher eccentricities and why stopped motion is never seen at the fovea. One cause of the motion slowing reported here might be that there were relatively few motion sensors tuned to the particular slow speed present in our stimuli at large eccentricities, so that a little adaptation quickly brought the motion below threshold. ${ }^{1}$

It has been shown that motion appears to slow down as luminance contrast decreases (Anstis, 2003; Stone \& Thompson, 1992; Thompson, 1982; Thompson \& Stone, 1997; Thompson, Stone, \& Swash, 1996). However, motion fading is unlikely to be due to decreases of perceived luminance contrast of the target stimulus following neuronal adaptation, because the apparent contrast of a stimulus does not appear to change upon or during motion fading. For example, the luminance contrast of the stimulus just before and just after illusory cessation of motion does not appear to brighten or dim. Moreover, the results from Experiment 4 (Figure 6) rule out the possibility that motion fading arises simply because of a decrease in perceived luminance contrast or brightness, because if a decrease of perceived luminance contrast after adaptation were the sole cause of motion fading, all the perceived luminances would have dropped to the lowest luminance that a stimulus needs to have in order to be perceived as moving, which was not found to be the case.

Motion fading appears to be an example of a diverse class of phenomena where a visual feature vanishes from consciousness although still present in the stimulus. Other examples of such visual fading from consciousness involve the subjective disappearance of a stationary object, as in the Troxler effect (Livingstone \& Hubel, 1987; Troxler, 1804) or motion-induced blindness (Bonneh, Cooperman, \& Sagi, 2001). It has been hypothesized (De Weerd, Gattass, Desimone, \& Ungerleider, 1995; Gerrits, De Haan, \& Vendrik, 1966; Gerrits \& Vendrik, 1970; Spillmann \& De Weerd, 2003) that during perceptual fading, the vanished target area is actively filled in with information from the background. In motion fading, however, only the motion component appears to vanish from consciousness, whereas the form component may remain visible. Because the form of the stimulus may remain visible, it is unlikely that information from the surround replaces the stimulus. Motion fading is therefore not likely to be reducible to other classes of visual fading/filling-in that involve the subjective disappearance of a stationary object. In particular, the filling-in mechanism proposed to underlie other examples of perceptual fading is unlikely to play a role in motion fading.

Instead, we report here evidence that motion fading likely results from the same neural adaptation over the same neural population as that giving rise to the MAE. A fascinating phenomen that is consistent with the hypothesis that motion fading and the MAE arise from a common neuronal mechanism is that physically stopping rotation during a period when the rotating stimulus subjectively appears to have stopped leads to apparent rotation in the direction opposite that of previous stimulus rotation, consistent with the direction of the MAE. We tested this hypothesis in Experiment 5 by showing that adapting to a motion pattern that could generate the MAE would facilitate motion fading on a subsequent motion pattern. This finding is quite similar to that in studies on motion threshold increase from preadaptation, as has been shown in the MAE literature (Raymond, 1994). In Experiment 6 , we found that (1) the duration of the MAE was positively correlated with the duration of time for which the counterclockwise rotating pattern was presented, and (2) for cases in which the counterclockwise rotating pattern had appeared to stop, when the pattern in fact physically stopped during subjective stopping, the duration of the MAE was constant. This presumably occurred because the level of neuronal adaptation had reached a maximum when the counterclockwise rotating pattern appeared to be stopped (i.e., when the adapted neurons no longer encoded the presence of a motion signal). Together, these results suggest that motion fading and MAE share a common neuronal adaptation mechanism.

The MAE occurs because of neural adaptation (i.e., modulation of gain control) near or at the input of MT (Kohn \& Movshon, 2003). Because our data imply that motion fading and the MAE occur because of adaptation in the same population of neurons, it follows that motion fading is also realized in the motion pathway at or near the input to area MT. Such neural adaptation is realized in the motion pathway, not the form pathway (Kohn \& Movshon, 2003). This is consistent with the phenomenology of motion fading, where only the motion component appears to vanish from consciousness, whereas the form component remains visible. From this, it can be concluded that the neural basis of conscious motion perception may be realized in a population of neurons that is different from that which realizes conscious form perception. In motion fading, the neuronal population in the motion pathway adapts to the point that a motion input falls below threshold, whereas the population in the form pathway continues to respond. This supports the notion that different aspects of conscious experience, such as form and motion, are realized in different populations of neurons (Zeki, 2007), which can be differentially adapted and, in principle, enhanced or eliminated, as through selective ablation. Certainly there are patients with akinetopsia who lack motion perception yet still see form (Zihl, von Cramon, Mai, \& Schmid, 1991). It is less clear whether patients who lack configural aspects of form perception, such as apperceptive agnosics, have intact motion processing (Hildebrandt, Schütze, Ebke, \& Spang, 2004). This stands to reason because many higher level aspects of motion perception involve the motion of 3-D shapes, which in the absence of shape perception would be lost. One prediction of a multiple-neural-population model of consciousness, however, would be that there should be a phenomenon, which we might call form fading, where the population of neurons underlying form perception has been "silenced," perhaps through adaptation, but the population of neurons underlying motion perception remains active. As far as we know, such a phenomenon, if it exists, remains to be discovered.

It has been argued that there are at least two types of MAEs: the static aftereffect, for which a static stimulus is used to test MAE, and the flicker aftereffect, for which a 
dynamic stimulus is used to test MAE (von Grünau, 1986). Moreover, it has been shown that the static aftereffect is tuned to temporal frequency (Over, Broerse, Crassini, \& Lovegrove, 1973; Pantle, 1974) and the flicker aftereffect is tuned to velocity (Ashida \& Osaka, 1995; Thompson, 1981). Looking at the present results, it appears that motion fading depends on both velocity and temporal frequency: TTS increases with increasing velocity and increasing temporal frequency. (In Experiment 3, we modulated spatial frequency while keeping drifting speed constant, which is equivalent to modulating temporal frequency.) However, since our stimuli and experimental design were not designed specifically for distinguishing these two types of MAE, we cannot conclude whether motion fading has more to do with the flicker or the static MAE.

Several phenomenally related illusions have been discovered lately and deserve to be mentioned. For example, a motion standstill phenomenon has been reported: When observers view color-defined moving stimuli, a quickly moving object appears to stand still, with its details clearly visible. (Cavanagh, Tyler, \& Favreau, 1984; Lu, Lesmes, \& Sperling, 1999; Mullen \& Boulton, 1992; Teller \& Lindsey, 1993). Although both the motion standstill phenomenon and motion fading appear to involve motion stopping, we believe that the two phenomena involve distinct mechanisms. In the motion standstill effect, apparent stopping might arise because of the motion system's failure to detect any motion signals (Lu et al., 1999); in motion fading, in contrast, apparent stopping arises, we argue, because of neuronal adaptation among motion-tuned neurons.

Another illusion that shares similarities with motion fading is the phenomenon of illusory motion reversals (also known as the wagon wheel illusion in continuous light) (Holcombe, Clifford, Eagleman, \& Pakarian, 2005; Kline, Holcombe, \& Eagleman, 2004, 2006; Purves, Paydarfar, \& Andrews, 1996; VanRullen, 2006, 2007; VanRullen, Reddy, \& Koch, 2005, 2006). It has been proposed that illusory motion reversals could reflect a motion-during effect (an aftereffect that would be visible even during physical motion; see Kline et al., 2004, 2006; Pakarian \& Yasamy, 2003). Although this motion-during-effect hypothesis has been challenged by VanRullen (2007), showing that motion aftereffects and illusory motion reversals are not always correlated, our present finding might be treated as a different example of such a motion-during effect, in that the effects of adaptation among motion-tuned neurons are visible even during physical motion.

That the form of the stimulus remains visible after motion stopping must mean that a different population of neurons subserves the conscious experience of visual form. Moreover, that the form does not get updated, often for several seconds during perceived motion stopping, suggests that the neurons subserving the conscious experience of a particular form at a particular location require input from the motion system in order to update form information. In the absence of such an update signal, the form perceived is the most recently updated percept, and changes in form or orientation that are present in the stimulus are not experienced. Indeed, when motion fading is broken, as by a small eye movement, the frozen form appears to move abruptly to its new form or orientation.

To conclude, our findings may be accounted for by a simple model that posits adaptation among neurons tuned to motion in the direction of the moving stimulus. When the speed of stimulus motion is very low (i.e., near threshold for motion-tuned cells), adaptation results in the loss of any motion signal in that direction, leading to perceived motion stopping. When the speed of stimulus motion is high, adaptation lowers but does not end neuronal responses that encode motion signal in that direction, leading to perceived motion slowing, even in the absence of complete motion fading. Thus, the MAE and motion fading may share an at least partially overlapping mechanism of neuronal adaptation among common motion-tuned units under prolonged exposure to motion in a given direction.

\section{AUTHOR NOTE}

This project was funded by NIH Grant R03 MH0609660-01 to P.U.T. We thank two anonymous reviewers for comments. Correspondence concerning this article should be addressed to P.-J. Hsieh, Brain and Cognitive Sciences, Massachusetts Institute of Technology, 77 Massachusetts Ave., 46-4141, Cambridge, MA 02139 (e-mail: pjh@mit.edu).

\section{REFERENCE}

Anstis, S. (1996). Adaptation to peripheral flicker. Vision Research, 36, 3479-3485.

Anstis, S. (2003). Moving objects appear to slow down at low contrasts. Neural Networks, 16, 933-938.

Ashida, H., \& OsaKa, N. (1995). Motion aftereffect with flickering test stimuli depends on adapting velocity. Vision Research, 35, 18251833.

Bonneh, Y. S., Cooperman, A., \& Sagi, D. (2001). Motion-induced blindness in normal observers. Nature, 14, 798-801.

Campbell, F. W., \& MafFeI, L. (1979). Stopped visual motion. Nature, 278, 192-193.

CAmpbell, F. W., \& MaffeI, L. (1981). The influence of spatial frequency and contrast on the perception of moving patterns. Vision Research, 21, 713-721.

Castet, E., Keeble, D. R., \& Verstraten, F. A. (2002). Nulling the motion aftereffect with dynamic random-dot stimuli: Limitations and implications. Journal of Vision, 2, 302-311.

Cavanagh, P., Tyler, C. W., \& Favreau, O. E. (1984). Perceived velocity of moving chromatic gratings. Journal of the Optical Society of America A, 1, 893-899.

CoHen, R. L. (1965). Adaptation effects and aftereffects of moving patterns viewed in the periphery of the visual field. Scandinavian Journal of Psychology, 6, 257-264.

De Weerd, P., Gattass, R., Desimone, R., \& Ungerleider, L. G. (1995). Responses of cells in monkey visual cortex during perceptual filling-in of an artifical scotoma. Nature, 377, 731-734.

Frome, F. S., MacLeod, D. I., Buck, S. L., \& Williams, D. R. (1981). Large loss of visual sensitivity to flashed peripheral targets. Vision Research, 21, 1323-1328.

Gerrits, H. J. M., De HaAn, B., \& Vendrik, A. J. H. (1966). Experiments with retinal stabilized images: Relations between the observations and neural data. Vision Research, 6, 427-440.

Gerrits, H. J. M., \& VendRIK, A. J. H. (1970). Simultaneous contrast, filling-in process and information processing in man's visual system. Experimental Brain Research, 11, 411-430.

Grunewald, A., \& Lankheet, M. J. M. (1996). Orthogonal motion after-effect illusion predicted by a model of cortical motion processing. Nature, 384, 358-360.

Hammett, S. T., \& Smith, A. T. (1990). Flicker adaptation in the periphery at constant perceived modulation depth. Perception, 19, 113-117. Harris, J. P., Calvert, J. E., \& Snelgar, R. S. (1990). Adaptation to 
peripheral flicker: Relationship to contrast detection thresholds. $\mathrm{Vi}$ sion Research, 30, 381-386.

Hildebrandt, H., Schütze, C., EbKe, M., \& Spang, K. (2004). Differential impact of parvocellular and magnocellular pathways on visual impairment in apperceptive agnosia? Neurocase, 10, 207-214.

Holcombe, A. O., Clifford, C. W., Eagleman, D. M., \& Pakarian, P. (2005). Illusory motion reversal in tune with motion detectors. Trends in Cognitive Sciences, 9, 559-560.

HsIEH, P.-J., \& TSE, P. U. (2007). Grouping inhibits motion fading by giving rise to virtual trackable features. Journal of Experimental Psychology: Human Perception \& Performance, 33, 57-63.

Hunzelmann, N., \& SPILlmanN, L. (1984). Movement adaptation in the peripheral retina. Vision Research, 24, 1765-1769.

Kline, K., Holcombe, A. O., \& Eagleman, D. M. (2004). Illusory motion reversal is caused by rivalry, not by perceptual snapshots of the visual field. Vision Research, 44, 2653-2658.

Kline, K., Holcombe, A. O., \& EAgleman, D. M. (2006). Illusory motion reversal does not imply discrete processing: Reply to Rojas et al. Vision Research, 46, 1158-1159.

KoHn, A., \& Movshon, J. A. (2003). Neuronal adaptation to visual motion in area MT of the macaque. Neuron, 39, 681-691.

Lichtenstein, M. (1963). Spatiotemporal factors in cessation of smooth apparent motion. Journal of the Optical Society of America A, 53, 304-306.

Livingstone, M. S., \& Hubel, D. H. (1987). Psychophysical evidence for separate channels for the perception of form, color, movement, and depth. Journal of Neuroscience, 7, 3416-3418.

Lu, Z. L., Lesmes, L. A., \& Sperling, G. (1999). Perceptual motion standstill in rapidly moving chromatic displays. Proceedings of the National Academy of Sciences, 96, 15374-15379.

MacKaY, D. M. (1982). Anomalous perception of extrafoveal motion. Perception, 11, 359-360.

Mullen, K. T., \& Boulton, J. C. (1992). Absence of smooth motion perception in color vision. Vision Research, 32, 483-488.

MuraKami, I., \& Shimojo, S. (1995). Modulation of motion aftereffect by surround motion and its dependence on stimulus size and eccentricity. Vision Research, 35, 1835-1844.

Over, R., Broerse, J., Crassini, B., \& Lovegrove, W. (1973). Spatial determinants of the aftereffect of seen motion. Vision Research, 13, 1681-1690.

Pakarian, P., \& Yasamy, M. T. (2003). Wagon-wheel illusion under steady illumination: Real or illusory? Perception, 32, 1307-1310.

PANTLE, A. (1974). Motion aftereffect magnitude as a measure of the spatiotemporal response properties of direction-sensitive analyzers. Vision Research, 14, 1229-1236.

Price, N. S. C., Greenwood, J. A., \& Ibbotson, M. R. (2004). Tuning properties of radial phantom motion aftereffect. Vision Research, 44, 1971-1979.

Purves, D., Paydarfar, J. A., \& Andrews, T. J. (1996). The wagon wheel illusion in movies and reality. Proceedings of the National Academy of Sciences, 93, 3693-3697.

RAYMOND, J. E. (1994). The effects of displacement size and frame duration on the motion-incoherence aftereffect. Perception, 23, 1203-1209.

Schieting, S., \& SpillmanN, L. (1987). Flicker adaptation in the peripheral retina. Vision Research, 27, 277-284.

Spillmann, L., \& De WeERD, P. (2003). Mechanisms of surface completion: Perceptual filling-in of texture. In L. Pessoa \& P. De Weerd (Eds.), Filling-in: From perceptual completion to cortical reorganization (pp. 81-105). Oxford: Oxford University Press.

Stone, L. S., \& Thompson, P. (1992). Human speed perception is contrast dependent. Vision Research, 32, 1535-1549.

Teller, D. Y., \& Lindsey, D. T. (1993). Motion at isoluminance: Motion dead zones in three-dimensional color space. Journal of the Optical Society of America A, 10, 1324-1331.

Thompson, P. (1981). Velocity after-effects: The effects of adaptation to moving stimuli on the perception of subsequently seen moving stimuli. Vision Research, 21, 337-345.

THOMPSON, P. (1982). Perceived rate of movement depends on contrast. Vision Research, 22, 377-380.
Thompson, P., \& Stone, L. S. (1997). Contrast affects flicker and speed perception differently. Vision Research, 37, 1255-1260.

Thompson, P., Stone, L. S., \& Swash, S. (1996). Speed estimates from grating patches are not contrast-normalized. Vision Research, 36, 667674.

TroxLER, D. (1804). Über das Verschwinden gegebener Gegenstände innerhalb unsers Gesichtskreises. In K. Himly \& J. A. Schmidt (Eds.), Ophthalmologisches Bibliothek (pp. 51-53) Jena: Fromman.

van DE Grind, W. A., KoenderinK, J. J., \& VAN Doorn, A. J. (1986). The distribution of human motion detector properties in the monocular visual field. Vision Research, 26, 797-810.

VAN de Grind, W. A., Lankheet, M. J., \& TaO, R. (2003). A gaincontrol model relating nulling results to the duration of dynamic motion aftereffects. Vision Research, 43, 117-133.

van de Grind, W. A., van der Smagt, M. J., \& Verstraten, F. A. (2004). Storage for free: A surprising property of a simple gain-control model of motion aftereffects. Vision Research, 44, 2269-2284.

van de Grind, W. A., van Hof, P., van der Smagt, M. J., \& VerStRaten, F. A. (2001). Slow and fast visual motion channels have independent binocular-rivalry stages. Proceedings of the Royal Society $B, \mathbf{2 6 8}, 437-443$.

van der Smagt, M. J., Verstraten, F. A. J., \& van de Grind, W. A. (1999). A new transparent motion aftereffect. Nature Neuroscience, 2, 595-596.

VANRULLEN, R. (2006). The continuous wagon wheel illusion is objectbased. Vision Research, 46, 4091-4095.

VANRULLEN, R. (2007). The continuous wagon wheel illusion depends on, but is not identical to neuronal adaptation. Vision Research, 47, 2143-2149.

VanRullen, R., Reddy, L., \& Koch, C. (2005). Attention-driven discrete sampling of motion perception. Proceedings of the National Academy of Sciences, 102, 5291-5296.

VanRullen, R., Reddy, L., \& Koch, C. (2006). The continuous wagon wheel illusion is associated with changes in electroencephalogram power at approximately $13 \mathrm{~Hz}$. Journal of Neuroscience, 26, 502-507.

Verstraten, F. A. J., van der Smagt, M. J., Fredericksen, R. E., \& VAN DE GRIND, W. A. (1999). Integration after adaptation to transparent motion: Static and dynamic test patterns result in different aftereffect directions. Vision Research, 39, 803-810.

Verstraten, F. A. J., van der Smagt, M. J., \& Van de Grind, W. A. (1998). Aftereffect of high-speed motion. Perception, 27, 1055-1066. VON GrÜNAU, M. W. (1986). A motion aftereffect for long-range stroboscopic apparent motion. Perception \& Psychophysics, 40, 31-38.

WoHLgEMUTH, A. (1911). On the after-effect of seen movement. British Journal of Psychology, Monograph Supplement, 1, 1-117.

WRIGHT, M. J. (1986). Apparent velocity of motion aftereffects in central and peripheral vision. Perception, 15, 603-612.

Zeki, S. (2007).The disunity of consciousness. Progress in Brain Research, 168, 11-18, 267-268.

Zihl, J., von Cramon, D., MaI, N., \& Schmid, C. (1991). Disturbance of movement vision after bilateral posterior brain damage: Further evidence and follow up observations. Brain, 114, 2235-2252.

\section{NOTE}

1. Alternatively, it is possible that this eccentricity effect is due to some kind of motion distortion of the comparison stimulus (for which the eccentricity varies just as much, due to its mirror location). For example, if briefly flashed (1-sec) motion pulses (see the Method section) are perceived to be faster than they truly are as one goes toward the periphery (e.g., due to the greater ratio of magnocellular inputs), they will be more adjusted, and it will seem as if the constant stimulus speed has decreased. Further experiments are required to test this possibility.

(Manuscript received January 31, 2008; revision accepted for publication November 12, 2008.) 